\title{
Rapid Semi-Quantitative Analysis of Formaldehyde in Food by Digital Image Colorimetry
}

\author{
Jinda Rungroadsri, Wadcharawadee Limsakul, Worawit Wongniramaikul, and Aree Choodum
}

\begin{abstract}
Digital image colorimetry was successfully applied for the rapid semi-quantitative analysis of formalin in fresh food. A built-in digital camera in mobile phone was used to image a colorimetric product of formaldehyde, while a custombuilt color analysis program was used to analyze the color intensity of the digital image. A colorimetric product (yellow) was obtained from the colorimetric reaction between the colorimetric reagents entrapped within a custom-built sensor and formaldehyde. A wide linear range $(1$ to $50 \mathrm{mg} / \mathrm{L})$ with good linearity (>0.99) was obtained for formaldehyde quantification. Good inter-day precision (1.30-4.37\%RSD) with low detection limit $(0.016 \mathrm{mg} / \mathrm{L})$ were obtained. The method was applied for semi-quantitative analysis of formaldehyde in fresh foods from fresh markets in Phuket. The concentrations of formaldehyde in the foods were lower than their natural occurrence.
\end{abstract}

Index Terms-Digital image colorimetry, formaldehyde, mobile phone, colorimetric sensor.

\section{INTRODUCTION}

Formaldehyde is a widely used industrial chemical due to its high reactivity and relatively low cost [1]. It is mainly used as a precursor in many industries such as textile, plastic, and wood industries [2]-[4]. It is also commonly used as a preservative in medical laboratories, mortuaries, and consumer products. Formaldehyde can cause severe health issues. It irritates to eyes and nose, damages central nervous system, and disorders immune system [1]. The International Agency for Research in Cancer (IARC) has classified it as carcinogenic to human.

Several inappropriate use of formaldehyde for extending food shelf-life has been reported in many countries including Thailand. For example, there was a contamination of formaldehyde in squid and prawn sold at a fresh market in Chiangmai [5]. The concentration of $18.87 \pm 1.38 \mathrm{ppm}$ and $19.32 \pm 1.70 \mathrm{ppm}$ were found in the samples which are higher than their natural occurrence (i.e. $1.8 \mathrm{ppm}$ in squid and 1.0$2.4 \mathrm{ppm}$ in prawn), [6]. The determination of formaldehyde in food is then required.

A wide range of instrumental methods has been reported for the determination of formaldehyde, e.g. gas chromatography [7], high performance liquid

Manuscript received January 14, 2017; revised May 9, 2017. This work was supported by Faculty of Technology and Environment, Prince of Songkla University, Phuket Campus.

The authors are with the Faculty of Technology and Environment, Prince of Songkla University, Phuket Campus, Phuket, 83120 Thailand (email: wadcharawadee.n@phuket.psu.ac.th, worawit.won@phuket.psu.ac.th, aree.c@phuket.psu.ac.th, jinda.r@phuket.psu.ac.th). chromatography [8], capillary electrophoresis [9], and sensors [10]. These methods need to be done in laboratory using expensive instruments.

Recently, a low-cost colorimetric method has been used in conjunction with digital image analysis as a real-time and rapid quantitative method for various analytes [11]-[13]. It is based on the analysis of RGB values (Red Green Blue basic color) from digital images of the colorimetric product. Due to the reflected light from the colorimetric product passes through RGB filters during imaging. This makes the light separated into three spectral ranges. The separated lights are detected and recorded as individual RGB values by the image sensor located behind each filter. The combination of recorded RGB data generates a final color digital image of the colorimetric product. Analysis of the digital images using color analysis program, e.g. Matlab [14], Kylix [15], Visual basic [16], or Adobe Photoshop [17] provided a recorded RGB value. These values can be used as the analytical data to produce a calibration graph for quantitative analysis of interested analyte.

In this work, a digital image colorimetry (DIC) was developed for a rapid semi-quantitative analysis of formaldehyde in foods from fresh markets in Phuket.

\section{EXPERIMENTAL}

\section{A. Colorimetris Test of Formaldehyde}

Formaldehyde solution (37\%w/v, Supelco Bellefonte, PA) was used to prepare a stock solution $(1000 \mathrm{mg} / \mathrm{L})$. Standard working solutions were prepared daily by diluting of the stock solution in ultrapure water (Barnstead EasyPure II, Thermo fisher scientific, $\mathrm{OH}$ ) to appropriate concentrations.

A custom-built formaldehyde test kit was used for colorimetric test of formaldehyde. It was developed based on the entrapment of colorimetric reagents within polymer matrix at the bottom of small sample vial $(2.5 \mathrm{~mL})$. Acetylacetone (Sigma-Aldrich, USA) in ammonium acetate (Sigma-Aldrich, USA) was used as a colorimetric reagent. Sample solution can be directly added into the test kit for colorimetric detection of formaldehyde.

\section{B. Photographic System and Procedure}

A custom-built photographic box [18] was used throughout the experiment. Three sample vials (sensors) were hung at the top of the box. The colorimetric product from the kits were imaged under reproducible illumination using a built-in camera of mobile phone (Samsung Galaxy Core II) which was put in the slot at the front wall of the box.

The RGB values of the digital images were analyzed using a custom-built RGB analysis program installed on the 
computer.

\section{Quantification of Formaldehyde}

Calibration curve for quantification of formaldehyde was investigated. A series of known concentration formaldehyde standard solutions was added into three sensors to produce color products. The products were photographed at 1 minute post reaction. RGB values from these images were then analyzed using a custom-built color analysis program. Ratios between intensity of blue and red $\left(I_{B} / I_{R}\right)$, blue and green $\left(I_{B} / I_{G}\right)$, and blue and total $\left(I_{B} / I_{\text {TOTAL }}\right)$ were plotted against the concentrations of formaldehyde solutions to establish a standard curve.

\section{Analytical Performance and Method Validation}

Sensitivity, linearity, linear range, limit of detection (LOD) and limit of quantification (LOQ) [19] were investigated. Precision was studied in terms of percentage relative standard deviation (\%RSD) for RGB values. The accuracy was evaluated by analyzing the known concentration of formaldehyde standard $(7.5 \mathrm{mg} / \mathrm{L})$ against the established standard curve.

\section{E. Real Sample Analysis}

Fresh foods from fresh markets in Phuket were tested using the formaldehyde sensor coupled with the DIC. The foods were immersed in water for 15 minutes. The water samples were then tested with formaldehyde sensor and DIC. The foods were washed further until clean and ready to cook. They were then immersed in water for further 15 minutes. The water samples were then tested as described.

\section{RESULTS AND DISCUSSION}

\section{A. Colorimetric Test of Formaldehyde.}

Due to custom-built formaldehyde sensor used in this work based on the Hantzsh reaction, yellow product (Fig. 1) was obtained [1], [20]-[22]. Colorimetric reagents consisted of acetylacetone, ammonium acetate, and acetic acid were entrapped within polymer matrix. Acetylacetone was used as $\beta$-diketone to be reacted with formaldehyde because it could produce more intense color product and react faster than methyl acetoacetate [1]. When formaldehyde sample solution was added into the sensor, the entrapped chemical reagents were dissolved allowing the colorimetric reaction occurred. Darker yellow products were obtained with increasing formaldehyde concentrations.

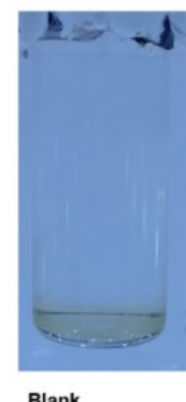

Blank

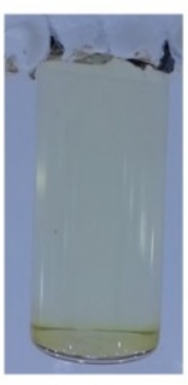

$10 \mathrm{mg} / \mathrm{L}$

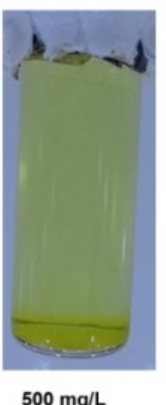

$500 \mathrm{mg} / \mathrm{L}$
Fig. 1. Color products from custom-built formaldehyde sensors.

\section{B. Digital Image Colorimetry for Formaldehyde}

Individual RGB values were obtained from systematic analysis of digital images of colorimetric products. Intensity of blue channel $\left(I_{B}\right)$ was higher than green $\left(I_{G}\right)$ and red $\left(I_{R}\right)$ channels at low concentrations of formaldehyde $(<25 \mathrm{mg} / \mathrm{L})$. Darker yellow products obtained at high concentrations $(>25$ $\mathrm{mg} / \mathrm{L}$ ) provided higher $I_{G}$ and $I_{R}$ than $I_{B}$. Intensities of red and green lights seemed to be constant with increasing the concentrations of formaldehyde, but $I_{B}$ was decreased (Table I). These indicated that the yellow products absorbed the light at the blue range $(400-500 \mathrm{~nm})$ which was in agreement with spectrophotometric results ( $\lambda_{\max }$ at $\left.462 \mathrm{~nm}\right)$. When more products produced at higher concentrations, the darker color products absorbed more blue light, thus provided less intensities.

TABLE I: INDIVIDUAL RGB INTENSITIES FROM YELLOW PRODUCTS AT VARIOUS CONCENTRATION OF FORMALDEHYDE

\begin{tabular}{cccc}
\hline \hline Concentration (mg/L) & $R$ (a.u.) & $G$ (a.u.) & $B$ (a.u.) \\
\hline 0 & 107.1 & 146.5 & 195.8 \\
1 & 123.8 & 138.3 & 158.5 \\
5 & 126.6 & 139.7 & 153.8 \\
10 & 130.7 & 140.7 & 146.9 \\
25 & 132.8 & 141.0 & 134.3 \\
50 & 130.4 & 140.6 & 110.5 \\
100 & 128.7 & 140.7 & 97.3 \\
250 & 126.6 & 139.9 & 82.5 \\
500 & 125.6 & 141.4 & 67.6 \\
\hline \hline
\end{tabular}

Total intensity ( $I_{\text {TOTAL }}: I_{R}+I_{G}+I_{B}$ ) was also investigated. It was found that $I_{\text {TOTAL }}$ decreased when the concentration of formaldehyde was increased as shown in Fig. 2. This was due to the higher amount of color product produced at higher concentration of formaldehyde could absorb more light.

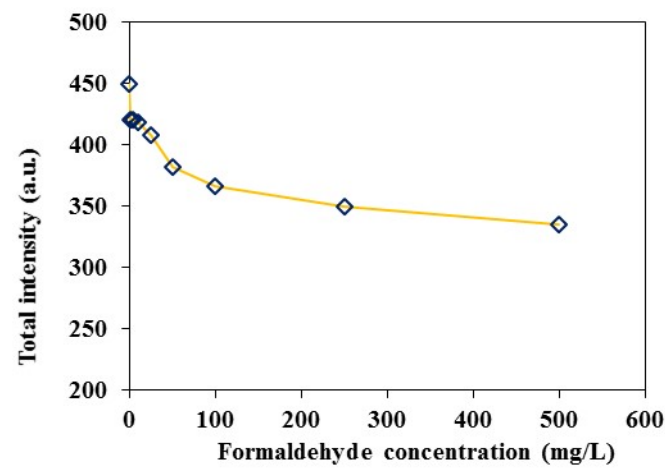

Fig. 2. The relationships between $I_{\text {TOTAL }}\left(I_{R}+I_{G}+I_{B}\right)$ with formaldehyde concentrations.

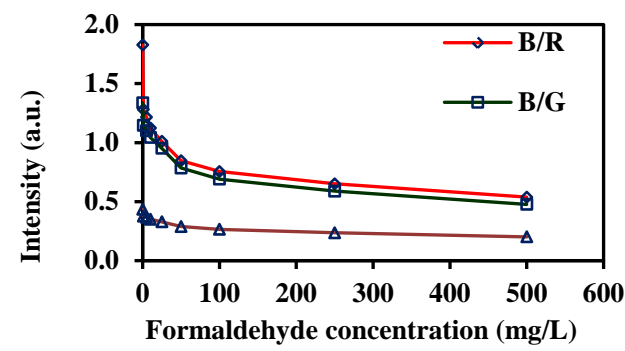

Fig. 3. The relationships between $I B / I R, I B / I G$, and IB/ITOTAL with formaldehyde concentrations.

The relationships of $I_{B} / I_{R}, I_{B} / I_{G}$, and $I_{B} / I_{\text {TOTAL }}$ with formaldehyde concentrations are shown in Fig. 3. $I_{B} / I_{R}$ was higher than $\mathrm{I}_{\mathrm{B}} / I_{G}$ and $I_{B} / I_{\mathrm{TOTAL}}$. When concentration of formaldehyde was increased, the $I_{B} / I_{R}, I_{B} / I_{G}$, and $I_{B} / I_{\text {TOTAL }}$ 
were decreased because of less reflected light from darker products.

The relationships of formaldehyde concentration and $I_{B} / I_{G}$, and $I_{B} / I_{\text {TOTAL }}$ in the range of 1 to $50 \mathrm{mg} / \mathrm{L}$ was founded to be linear with linearity of 0.99 , and 0.9874 , respectively (Fig. 4).

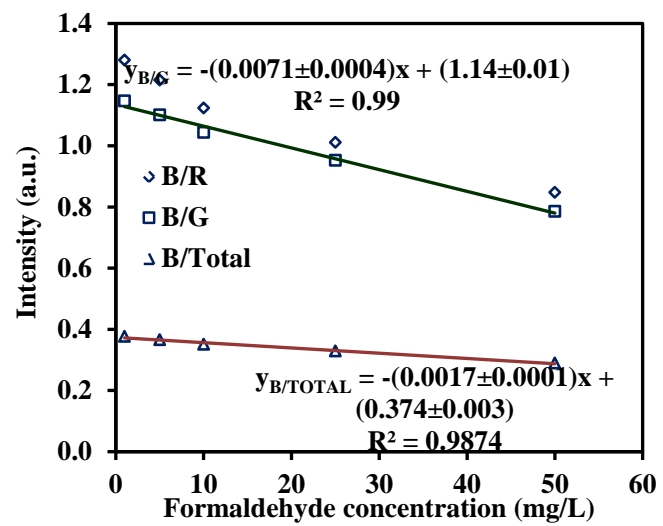

Fig. 4. The relationships between $I B / I R, I B / I G$, and $I B / I T O T A L$ with formaldehyde concentrations in the range of 1 to $50 \mathrm{mg} / \mathrm{L}$.

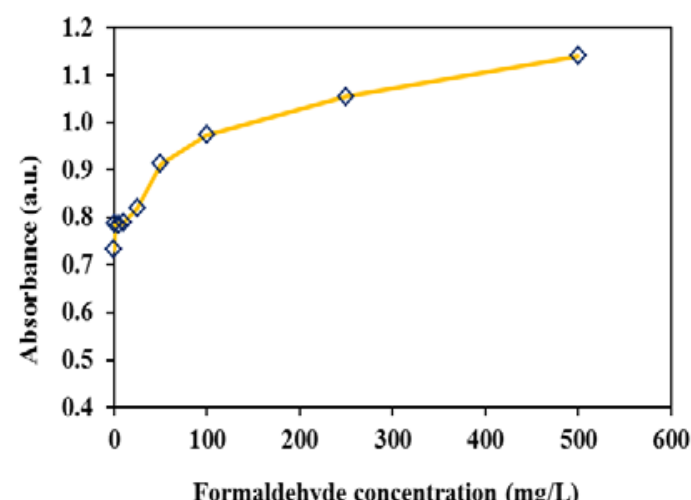

Fig. 5. The relationships between ATOTAL $(A R+A G+A B)$ with formaldehyde concentrations.

TABLE II: INDIVIDUAL AND TOTAL RGB ABSORBANCE FROM YELLOW PRODUCTS AT VARIOUS CONCENTRATION OF FORMALDEHYDE

\begin{tabular}{ccccc}
\hline $\begin{array}{c}\text { Concentration } \\
(\mathrm{mg} / \mathrm{L})\end{array}$ & $R$ (a.u.) & $G$ (a.u.) & $B$ (a.u.) & Total (a.u.) \\
\hline 0 & 0.38 & 0.24 & 0.11 & 0.73 \\
1 & 0.31 & 0.27 & 0.21 & 0.79 \\
5 & 0.30 & 0.26 & 0.22 & 0.78 \\
10 & 0.29 & 0.26 & 0.24 & 0.79 \\
25 & 0.28 & 0.26 & 0.28 & 0.82 \\
50 & 0.29 & 0.26 & 0.36 & 0.91 \\
100 & 0.30 & 0.26 & 0.42 & 0.97 \\
250 & 0.30 & 0.26 & 0.49 & 1.05 \\
500 & 0.31 & 0.26 & 0.58 & 1.14 \\
\hline \hline
\end{tabular}

When the molecular absorption of the yellow products was calculated [15], [16], the blue channel $\left(A_{B}\right)$ which was increased with concentration of formaldehyde revealed the highest level of absorbance (Table II). This was a good agreement with spectrophotometric result which revealed the maximum absorption of the product in the blue range of $462 \mathrm{~nm}$.

Total absorbance ( $A_{\text {TOTAL }}: A_{R}+A_{G}+I_{B}$ ) was also investigated. $\mathrm{A}_{\text {TOTAL }}$ increased when the concentration of formaldehyde was increased (Fig. 5). The relationships of $A_{B} / A_{R}, \quad A_{B} / A_{G}, \quad$ and $A_{B} / A_{\text {TOTAL }}$ with formaldehyde concentrations (Fig. 6) revealed that $A_{B} / A_{G}$ was higher than
$A_{B} / A_{R}$ and $A_{B} / A_{\text {TOTAL }}$. When concentration of formaldehyde was increased, the $A_{B} / A_{G}, A_{B} / A_{\text {TOTAL }}$, and $A_{B} / A_{R}$ were increased because the darker products absorbed more light. The relationships of formaldehyde concentration and $A_{B} / A_{G}$, and $A_{B} / A_{R}$ in the range of 1 to $50 \mathrm{mg} / \mathrm{L}$ was founded to be linear with linearity of 0.9965 , and 0.9888 , respectively (Fig. 7).

\section{Calibration Curve for Quantification of Formaldehyde}

The linear relationships of formaldehyde concentration and $I_{B} / I_{G}, I_{B} / I_{\text {TOTAL }}, A_{B} / A_{G}, A_{B} / A_{R}$ in the range of 1 to $50 \mathrm{mg} / \mathrm{L}$ could be used as standard curves for quantification of formaldehyde. However, the relationships of $A_{B} / A_{G}$ revealed better sensitivity and linearity, it was selected for quantitative analysis of formaldehyde in real sample. The calibration equation as in (1) was used.

$$
y=-(0.0125 \pm 0.0004) x+(0.78 \pm 0.01)
$$

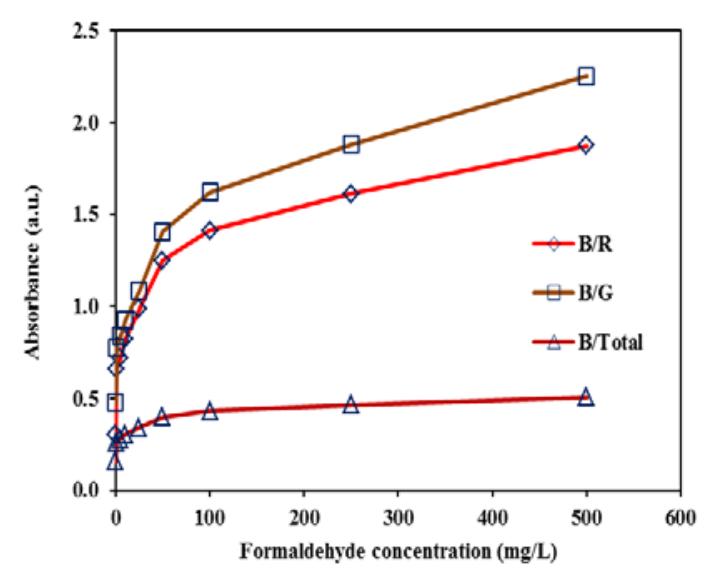

Fig. 6. The relationships between $A_{B} / A_{R}, A_{B} / A_{G}$, and $A_{B} / A_{\text {TOTAL }}$ with formaldehyde concentrations.

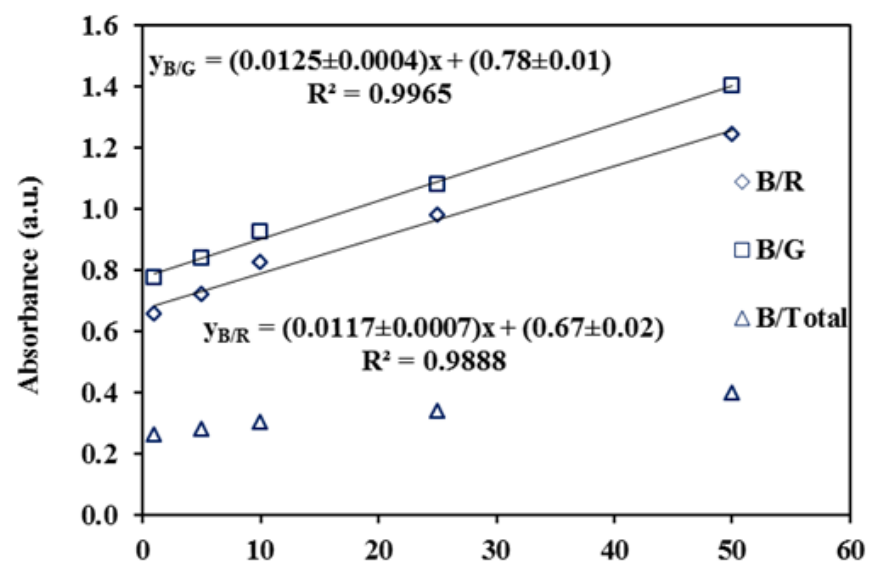

Formaldehyde concentration $(\mathbf{m g} / \mathbf{L})$

Fig. 7. The relationships between $A_{B} / A_{R}, A_{B} / A_{G}$, and $A_{B} / A_{\text {TOTAL }}$ with formaldehyde concentrations.

\section{Analytical Performance and Method Validation}

Analysis of formaldehyde using a custom-built formaldehyde sensor and DIC provided a linear quantification equation in the range of 1 to $50 \mathrm{mg} / \mathrm{L}$ with good linearity $(>0.99)$. The developed method provided sensitivity of $0.0125 \pm 0.0004$ a.u./(mg/L). LOD and LOQ of 0.016 and $0.053 \mathrm{mg} / \mathrm{L}$ were obtained indicating a good performance of the method for quantification of formaldehyde in food. Good intra-day (0.28-2.87\%RSD) 
and inter-day precisions (1.30-4.37\%RSD; $n=3)$ were obtained. When control samples of $7.5 \mathrm{mg} / \mathrm{L}$ were tested, the formaldehyde concentration of $6.5 \mathrm{mg} / \mathrm{L}$ was quantified indicating a good accuracy. These results indicated that the use of DIC together with custom-built formaldehyde sensor could be used for quantification of formaldehyde in food. The method made a quantification of formaldehyde easier and cheaper because it required only a daily used mobile phone compared to other expensive laboratory instruments.

\begin{tabular}{ll}
\multicolumn{2}{l}{ TABLE III: CONCENTRATION OF FORMALDEHYDE IN REAL SAMPLES } \\
\hline \hline Sample & Concentration of formaldehyde $(\mathrm{mg} / \mathrm{kg})$ \\
\hline Tomato & $0.1-0.7$ \\
Shred ginger & $0.04-0.1$ \\
Jew's ear mushroom & $0.5-0.7$ \\
Shrimp & $0.5-0.6$ \\
Fish & $0.3-0.4$ \\
Squid & Non detectable \\
\hline \hline
\end{tabular}

\section{E. Real Sample Analysis}

Fresh food samples including tomato, shred ginger, jew's ears mushroom, shrimp, fish, and squid from fresh markets in Phuket were tested using the developed method. Formaldehyde concentrations in a range of 0.04 to 0.7 $\mathrm{mg} / \mathrm{kg}$ were obtained (Table III). The highest formaldehyde concentrations were found in tomato and mushroom samples in the ranges of 0.1 to 0.7 , and 0.5 to $0.7 \mathrm{mg} / \mathrm{kg}$, respectively These quantified concentrations were lower than their natural occurrence.

\section{CONCLUSION}

A digital image colorimetry (DIC) was successfully developed for a rapid quantification of formaldehyde in fresh foods from fresh markets in Phuket. The analytical performance and method validation were found to be effective for determination of formaldehyde in foods. Analysis of fresh foods collected from fresh markets in Phuket showed lower concentration of formaldehyde than their natural occurrence.

\section{REFERENCES}

[1] O. Bunkoed, F. Davis., P. Kanatharana, P. Thavarungkul, and S. P. J. Higson, "Sol-gel based sensor for selective formaldehyde determination,” Anal. Chim. Acta, vol. 659, pp. 251-257, 2010.

[2] M. Guru, S. Tekeli, and I. Bilici, "Manufacturing of ureaformaldehyde-based composite particleboard from almond shell," Materials \& Design, vol. 27, pp. 1148-1151, 2006.

[3] S. Kim, "Environment-friendly adhesives for surface bonding of wood-based flooring using natural tannin to reduce formaldehyde and TVOC emission,” Bioresource Technol., vol. 100, pp. 744-748, 2009.

[4] S. Halvarsson, H. Edlund, and M. Norgren, "Properties of mediumdensity fibreboard (MDF) based on wheat straw and melamine modified urea formaldehyde (UMF) resin," Ind. Crops Prod., vol. 28, pp. 37-46, 2008.

[5] IARC, "Monographs on the evaluation of carcinogenic risks to humans, formaldehyde, 2-Butoxyethanol and 1-tert-Butoxy-2propanol," International Agency for Research on Cancer, Lyon, France, vol. 88, 2004.

[6] Clary and J. B. Sullivan, Formaldehyde, in Clinical Environmental Health and Toxic Exposures, Lippincott Williams and Wilkins, Philadelphia, p. 1008, 2001.

[7] T. Dumas, "Determination of formaldehyde in air by gas chromatography,” J. Chrom. A., vol. 247, pp. 289-295, 1982.
[8] A. Buldt, R. Lindahl, J.-O. Levin, and U. Karst, "A diffusive sampling device for the determination of formaldehyde in air using $\mathrm{N}$ methyl-4-hydrazino-7-nitrobenzofurazan (MNBDH) as reagent,” $J$. Environ, Monit., vol. 1, pp. 39-43, 1999.

[9] F. R. Rocha, G. H. G. Coelho, M. L. A. Lopes, L. F. R. Carvalho, J. A. Fracassi da Silva, C. L. do Lago, and I. G. R. Gutz, "Environmental formaldehyde analysis by active diffusive sampling with a bundle of polypropylene porous capillaries followed by capillary zone electrophoretic separation and contactless conductivity detection," Talanta, vol.76, pp. 271-275, 2008.

[10] J. A. Dirksen, K. Duval, and T. A. Ring, "NiO thin-film formaldehyde gas sensor," Sensor Actuat B-Chem, vol. 80, pp. 106-115, 2001.

[11] A. Choodum, P. Kanatharana, W. Wongniramaikul, and N. N. Daeid, "Rapid quantitative colourimetric tests for trinitrotoluene (TNT) in soil," Forensic Sci. Int., vol. 222, pp. 340-345, 2012.

[12] A. Choodum, K. Parabun, N. Klawach, N. N. Daeid, P. Kanatharana, and W. Wongniramaikul, "Real time quantitative colourimetric test for methamphetamine detection using digital and mobile phone technology,” Forensic Sci. Int., vol. 235, pp. 8-13, 2014.

[13] A. Choodum, P. Kanatharana, W. Wongniramaikul, and N. N. Daeid, "Using the iPhone as a device for a rapid quantitative analysis of trinitrotoluene in soil,” Talanta, vol. 115, pp. 143-149, 2013.

[14] A. Lopez-Molinero, D. Linan, D. Sipiera et al., "Chemometric interpretation of digital image colorimetry. Application for titanium determination in plastics,” Microchem. J., vol. 96, pp. 380-385, 2012.

[15] E. D. N. Gaiao, V. L. Martins, W. D. S. Lyra et al., "Digital imagebased titrations,” Anal. Chim. Acta, vol. 570, pp. 283-290, 2006.

[16] N. Maleki, A. Safavi, and F. Sedaghatpour, "Single-step calibration, prediction and real samples data acquisition for artificial neural network using a CCD camera,” Talanta, vol. 64, pp. 830-835, 2004.

[17] A. Choodum and N. N. Daeid, "Rapid and semi-quantitative presumptive tests for opiate drugs," Talanta, vol. 86, pp. 284-292, 2011.

[18] A. Choodum, P. Kanatharana, W. Wongniramaikul, and N. N. Daeid, "Poly vinyl alcohol cryogel as a selective test kit for pre and post blast trinitrotoluene,” Sensors and Actuators B: Chemical, vol. 222, pp. 654-662, 2016.

[19] J. N. Miller and J. C. Miller, Statistic and Chemometric for Analytical Chemistry, 5th ed., Essex: Pearson Education Limited, 2005.

[20] T. Nash, "The colorimetric estimation of formaldehyde by means of the Hantzsch reaction," Biochemistry, vol. 55, pp. 416-421, 1953.

[21] S. Belman, "The fluorometric determination of formaldehyde," Anal.Chim. Acta, vol. 29, pp. 120-126, 1963.

[22] Y. Y. Maruo, J. Nakamura, and M. Uchiyama, "Development of formaldehyde sensing element using porous glass impregnated with ß-diketone,” Talanta, vol. 74, pp. 1141-1147, 2008.

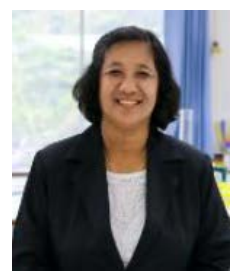

Jinda Rungroadsri was born in 1970. She received a bachelor of education in computer from Phuket Rajabhat University, Thailand.

She has been working at Prince of Songkla University, Phuket Campus, Thailand as a senior laboratory technician.

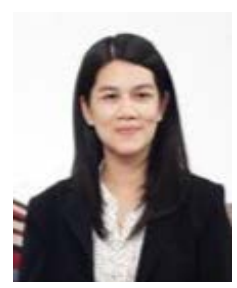

Wadcharawadee Limsakul was born in Yala Province, South of Thailand on August 15, 1979. She received a doctor of philosophy degree in bioengineering from The University of Queensland, Australia in 2010. In 2004 she obtained a master of science degree in polymer science from Chulalongkorn University, Thailand. She also got a bachelor of science degree in chemistry from Prince of Songkla University, Thailand in 2001. She has been working at Prince of Songkla University, Phuket Campus, Thailand since 2008 as a Lecturer. Her publications include B. Rai, W. Noohom, P. H. Kithva, L. Grondahl, and M. Trau, "Bionanohydroxyapatite/poly(3hydroxybutyrate-co-3-hydroxyvalerate) composites with improved particle dispersion and superior mechanical properties," Chemistry of Materials, vol. 20, pp. 2802-2808, 2008; W. Noohom, K. S. Jack, D. Martin, and M. Trau, "Understanding the roles of nanoparticle dispersion and polymer crystallinity in controlling the mechanical properties of HA/PHBV nanocomposites," Biomedical Materials, vol. 4, p. 015003, 2009; and A. Choodum, K. Malathong, N. NicDaeid, W. Limsakul, and W. Wongniramaikul, "A cost effective hydrogel test kit for pre and post blast trinitrotoluene," Forensic Science International, vol. 266, pp. 202-208, 
2016 .

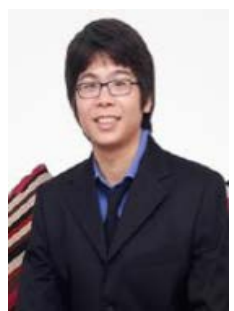

Worawit Wongniramaikul was born in 1979. He received a doctor of philosophy in environmental management from Chulalongkorn University, Thailand in 2007. He has been working at Prince of Songkla University, Phuket Campus, Thailand since 2007. He is currently an assistant professor. His recently publications is A. Choodum, K. Malathong, N. NicDaeid, W. Limsakul, and W. Wongniramaikul, "A cost effective hydrogel test kit for pre and post blast trinitrotoluene", Forensic Science International, vol. 266, pp. 202-208, 2016.

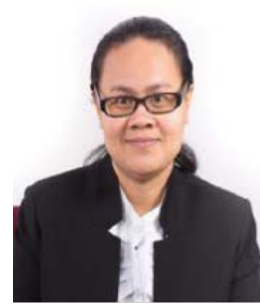

Aree Choodum was born in 1979. She received a doctor of philosophy in chemistry from Prince of Songkla University, Thailand in 2012. She has been working at Prince of Songkla University, Hat Yai Campus, Thailand since 2012. In 2014 she moved to Phuket Campus. She is currently an associate professor at the Faculty of Technology and Environment. Her recently publications is A. Choodum, K. Malathong, N. NicDaeid, W. Limsakul, and W. Wongniramaikul, "A cost effective hydrogel test kit for pre and post blast trinitrotoluene,” Forensic Science International, vol. 266, pp. 202-208, 2016. 